BULLETIN OF THE

AMERICAN MATHEMATICAL SOCIETY

Volume 80, Number 6, November 1974

\title{
A GENERAL THEORY OF IDENTITIES OF THE ROGERS-RAMANUJAN TYPE
}

BY GEORGE E. ANDREWS ${ }^{1}$

1. Introduction. This paper is a direct successor to [8], "Partition identities", henceforward referred to as PI. The lecture presented under the title of the present paper [9] can be roughly broken into three parts. Part 1 was a historical survey; Part 2 presented the basic elements of the theory of partition ideals and discussed partition ideals of order 1 since they possess rather elegant properties that are easily developed. Both Parts 1 and 2 essentially appear in $\S \S 1-3$ of PI. Part 3 (of [9]) was devoted to a discussion of how the more difficult questions in the theory of partition identities might be attacked. In $\$ 2$ we shall present a resumè of the elementary definitions and results for partition ideals. In $\$ 3$ below, we shall present in detail the discussion of the Rogers-Ramanujan identities that made up [9, Part 3]. It is now possible to provide a substantial partial answer to the first of the two main questions raised in $\$ 3$, and this result will be presented as Theorem 4.1 in $\$ 4$. $\$ 5$ will outline how the ideas involved in the proof of Theorem 4.1 have extended our knowledge of the general Rogers-Ramanujan theorem [6], [14]. In $\S 6$, we shall briefly survey the analytic results related to the second question raised in $\$ 3$. We conclude with a look at the open problems in this subject.

2. Partition ideals. All of the results in this section are presented (and proved) in PI. We shall, therefore, omit proofs and shall state only those results that are essential to the developments that follow. The lattice-theoretic definitions may be found in [19, Chapter 1].

Let $\mathscr{S}$ denote the set of all sequences $\left\{f_{i}\right\}_{i=1}^{\infty}$ (more briefly $\left\{f_{i}\right\}$ ), where each $f_{i}$ is a nonnegative integer and where only finitely many $f_{i}$ are nonzero. Then $\mathscr{S}$ forms a distributive lattice under the partial ordering $\left\{f_{i}\right\} \leqq\left\{g_{i}\right\}$ whenever $f_{i} \leqq g_{i}$ for each $i$. Furthermore the functions \# and $\sigma: \mathscr{S} \rightarrow N$ (where $N$ is the set of nonnegative integers) given by \# $\left(\left\{f_{i}\right\}\right)=$ $\sum f_{i}, \sigma\left(\left\{f_{i}\right\}\right)=\sum f_{i} \cdot i$ are positive valuations on $\mathscr{S}$. We let $\mathbf{0}=\{0,0,0, \cdots\}$ denote the constant sequence of zeros.

An expanded version of an invited address delivered before the 77th Summer Meeting of the Society at Dartmouth College, Hanover, New Hampshire on September 1, 1972; received by the editors February 9, 1974.

AMS (MOS) subject classifications (1970). Primary 10A45, 33A30; Secondary 05A15, 05A19.

Key words and phrases. Partitions, partition identities, Rogers-Ramanujan identities, generating functions.

${ }^{1}$ Partially supported by National Science Foundation Grant GP-23774. 
In intuitive terms, the sequences $\left\{f_{i}\right\}$ that make up $\mathscr{S}$ may each be thought of as defining a partition of a nonnegative integer where $f_{i}$ gives the number of times $i$ appears as a part. The function $\sigma$ maps each sequence $\left\{f_{i}\right\}$ onto the number being partitioned, and $\#\left(\left\{f_{i}\right\}\right)$ is the number of parts of the partition.

Recall now that a semi-ideal $J$ in a lattice $L$ is a subset of $L$ such that whenever $a \in J, x \in L$, and $x \leqq a$, then $x \in J[19$, p. 56].

Definition 1. A nonempty semi-ideal in the lattice $\mathscr{S}$ is called a partition ideal.

Definition 2. If $C$ is a partition ideal (in $\mathscr{S}$ ), we say that $p(C ; n)$ is the $C$-partition function if for each $n, p(C ; n)$ denotes the cardinality of the set $\left\{\left\{f_{i}\right\} \in C \mid \sigma\left(\left\{f_{i}\right\}\right)=n\right\}$.

Definition 3. We say two partition ideals $C_{1}$ and $C_{2}$ are equivalent (or partition-theoretically equivalent, or PT-equivalent) if for each nonnegative integer $n, p\left(C_{1} ; n\right)=p\left(C_{2} ; n\right)$. We shall write $C_{1} \sim^{\mathrm{PT}} C_{2}$.

Fundamental Problem. Fully describe the equivalence classes of partition ideals under the equivalence $\sim$ PT .

Definition 4. We say that a partition ideal $C$ has order $k$ provided $k$ is the least integer such that whenever $\left\{f_{i}\right\} \notin C$, there exists $m$ such that $\left\{f_{i}^{\prime}\right\} \notin C$, where

$$
\begin{aligned}
f_{i}^{\prime} & =f_{i}, \quad i=m, m+1, \cdots, m+k-1, \\
& =0, \quad \text { otherwise. }
\end{aligned}
$$

Intuitively, the assertion that $C$ is of order $k$ makes explicit the idea that if $\pi \in \mathscr{S}$, then summands of $\pi$ at least $k$ units apart cannot "interact" to affect whether $\pi \in \mathscr{S}$.

SeCond Problem. Fully describe the equivalence classes of partition ideals that contain a partition ideal of order 1 under the equivalence $\sim^{\text {PT }}$.

The partition ideals of order 1 have especially nice properties and are extremely amenable to various analytic and combinatorial techniques. The following results make this assertion abundantly obvious. The first result shows that if $C$ is of order 1 then the generating function for the $C$-partition function is an infinite product.

Theorem 2.1 [8, P. 20, Theorem 1]. Let $C$ be a partition ideal of order 1. Let $d_{l}=\sup _{\left\{f_{2}\right\} \in C} f_{l}$. Then for $|q|<1$,

$$
\sum_{n \geqq 0} p(C ; n) q^{n}=\prod_{l=1 ; d_{l}<\infty}^{\infty}\left(1-q^{l\left(d_{l}+1\right)}\right) / \prod_{l=1}^{\infty}\left(1-q^{l}\right) .
$$

The ideals of $\mathscr{S}$ are the partition ideals $C$ that are closed under union $\left[19\right.$, p. 56], where $\left\{f_{i}\right\} \cup\left\{g_{i}\right\}=\left\{\max \left(f_{i}, g_{i}\right)\right\}$. 
TheOREm 2.2 [8, P. 21, TheOREM 2]. The ideals in $\mathscr{S}$ are the partition ideals of order 1 .

Finally Theorem 2.1 implies a simple computational device for determining the equivalence of two partition ideals of order 1.

TheOREM 2.3 [8, P. 22, TheOREM 3]. Let $C$ be a partition ideal of order 1 and let the $d_{l}$ be defined as in Theorem 2.1. Let $C^{\prime}$ be a second partition ideal of order 1 with associated $d_{l}^{\prime}$. Then $C \sim{ }^{\mathrm{PT}} C^{\prime}$ if and only if the two multisets (i.e. sets with possible multiplicities of elements; see [29, p. 22] for detailed description of multisets) $\left\{j\left(d_{j}+1\right)\right\}_{d_{j}<\infty}$ and $\left\{j\left(d_{j}^{\prime}+1\right)\right\}_{d_{j}^{\prime<\infty}}$ are identical.

Theorem 2.3 provides us with the type of characterization one might possibly hope for in answer to the Fundamental Problem. As was shown in $\S 3$ of PI, questions restricted to possible equivalence of partition ideals of order 1 are extremely routine in virtue of Theorem 2.3. A theorem that dropped the restriction to order 1 but was similar in character to Theorem 2.3 in which whether or not $C \sim^{\mathrm{PT}} C^{\prime}$ could be answered by an algorithm that utilized certain easily ascertainable parameters of each partition ideal would certainly constitute an adequate answer to the Fundamental Problem.

3. The Rogers-Ramanujan identities. Our object now is to analyze one of the classical proofs of the Rogers-Ramanujan identities [31] using the terminology and approach of $\$ 2$. As a result of our analysis we shall pose two questions that appear to hold reasonable promise for attacking the Fundamental Problem (and the Second Problem) described in $\S 2$.

THEOREM 3.1. If $\mathscr{R}=\left\{\left\{f_{i}\right\} \in \mathscr{S} \mid f_{i}+f_{i+1} \leqq 1\right\}$ and $\mathscr{T}=\left\{\left\{f_{i}\right\} \in \mathscr{S} \mid f_{i}>0\right.$ implies $i \equiv 1,4(\bmod 5)\}$, then

$$
\mathscr{R} \sim \text { PT } \mathscr{T} .
$$

REMARK. This is the first of the Rogers-Ramanujan identities written in the language of partition ideals. We point out that the condition " $f_{i}+f_{i+1} \leqq 1$ " that defines $\mathscr{R}$ merely insures that in the partition $\sum_{i=1}^{\infty} f_{i} \cdot i=\sigma\left(\left\{f_{i}\right\}\right)$, no part repeats and no consecutive integers appear. Hence $p(\mathscr{R} ; n)$ is the number of partitions of $n$ in which the difference between parts is at least 2 . On the other hand if $\left\{f_{i}\right\} \in \mathscr{T}$, then $\sum_{i=1}^{\infty} f_{i} \cdot i$ is a partition in which only parts $\equiv 1,4(\bmod 5)$ may appear.

Thus Theorem 3.1 asserts that the partitions of each integer $n$ in which the difference between parts is at least 2 are equinumerous with the partitions of $n$ into parts $\equiv 1$ or $4(\bmod 5)$. This is the standard numbertheoretic formulation of the first Rogers-Ramanujan identity. 
SKETCH OF PROOF OF THEOREM 3.1. We begin by considering

$$
f_{\mathscr{R}}(x ; q) \equiv f_{\mathscr{R}}(x) \equiv \sum_{\left\{f_{\imath}\right\} \in \mathscr{R}} x^{\sum f_{i}} q^{\Sigma f_{i} \cdot i}
$$

a double series convergent at least for $|q|<1,|x|<|q|^{-1}$. Thus

$$
f_{\mathscr{R}}(1)=\sum_{n \geqq 0} p(\mathscr{R} ; n) q^{n}
$$

and, in fact, the coefficient of $x^{m} q^{n}$ in (3.1) is the number of partitions of $n$ belonging to $\mathscr{R}$ that have exactly $m$ parts.

Now

where

$$
\mathscr{R}=\mathscr{R}_{1} \cup \mathscr{R}_{2},
$$

$$
\mathscr{R}_{1}=\left\{\left\{f_{i}\right\} \in \mathscr{R} \mid f_{1}=0\right\}, \quad \mathscr{R}_{2}=\left\{\left\{f_{i}\right\} \in \mathscr{R} \mid f_{1}=1\right\} .
$$

By the definition of $\mathscr{R}$ it is clear that

$$
\begin{aligned}
& \mathscr{R}_{1}=\left\{\left\{f_{i}^{\prime}\right\} \mid f_{i}^{\prime}=f_{i-1}, i \geqq 2, f_{1}^{\prime}=0 \text { for some }\left\{f_{i}\right\} \in \mathscr{R}\right\} \\
& \mathscr{R}_{2}=\left\{\left\{f_{i}^{\prime \prime}\right\} \mid f_{i}^{\prime \prime}=f_{i-2}, i \geqq 3, f_{1}^{\prime \prime}=1, f_{2}^{\prime \prime}=0 \text { for some }\left\{f_{i}\right\} \in \mathscr{R}\right\} .
\end{aligned}
$$

Hence since $\mathscr{R}_{1} \cap \mathscr{R}_{2}=\varnothing$,

$$
\begin{aligned}
& f_{\mathscr{R}}(x)=\sum_{\left\{f_{\imath}\right\} \in \mathscr{R}} x^{\Sigma f_{i}} q^{\Sigma f_{2} i}=\left(\sum_{\left\{f_{\imath}\right\} \in \mathscr{R}_{1}}+\sum_{\left\{f_{i}\right\} \in \mathscr{R}_{2}}\right) x^{\Sigma f_{i}} q^{\Sigma_{f_{i} \cdot i}} \\
& =\sum_{\left\{f_{2}\right\} \in \mathscr{R}}\left(x^{\sum f_{l-1}} q^{\sum f_{l-1} i}+x^{1+\sum f_{i-2}} q^{1+\sum f_{l-2} i}\right) \\
& =\sum_{\left\{f_{i}\right\} \in \mathscr{R}}\left(x^{\Sigma f_{i}} q^{\sum f_{\imath}(i+1)}+x q x^{\Sigma f_{i}} q^{\Sigma f_{i}(i+2)}\right) \\
& =f_{\mathscr{R}}(x q)+x q f_{\mathscr{R}}\left(x q^{2}\right) .
\end{aligned}
$$

Now it is possible to prove in a completely elementary manner (coefficient comparison) that there is only one function $F(x)$ that is analytic in $x$ around $x=0$ and satisfies

$$
\begin{gathered}
F(0)=1, \\
F(x)=F(x q)+x q F\left(x q^{2}\right) .
\end{gathered}
$$

Clearly $f_{\mathscr{R}}(x)$ is such a function. On the other hand, ingenious but elementary arguments involving only the rearrangements of series [31] allow one to establish that if

$$
\phi(x)=\sum_{n \geqq 0} \frac{(-1)^{n} x^{2 n} q^{n(5 n+1) / 2}\left(1-x^{2} q^{4 n+2}\right)}{(1-q)\left(1-q^{2}\right) \cdots\left(1-q^{n}\right)\left(1-x q^{n+1}\right)\left(1-x q^{n+2}\right) \cdots},
$$

then $\phi(0)=1, \phi(x)$ is analytic in $x$ for $|q|<1,|x|<|q|^{-1}$, and $\phi(x)$ satisfies (3.4). 
Therefore $\phi(x)=f_{\mathscr{R}}(x)$ for $|q|<1,|x|<|q|^{-1}$. Hence

$$
\begin{aligned}
\sum_{n \geqq 0} p(\mathscr{R} ; n) q^{n} & =f_{\mathscr{R}}(1)=\phi(1) \\
& =\sum_{n \geqq 0}(-1)^{n} q^{n(5 n+1) / 2}\left(1-q^{4 n+2}\right) / \prod_{n=1}^{\infty}\left(1-q^{n}\right) \\
& =\prod_{n=0}^{\infty} \frac{1}{\left(1-q^{5 n+1}\right)\left(1-q^{5 n+4}\right)} \quad \text { (by Jacob's identity } \\
& =\sum_{n \geqq 0} p(\mathscr{T} ; n) q^{n} \quad(\text { py Theorem } 2.1) .
\end{aligned}
$$

Comparing coefficients in the extremes of this string of equations we see that $p(\mathscr{R} ; n)=p(\mathscr{T} ; n)$ for each $n$, i.e. $\mathscr{R} \sim^{\mathrm{PT}} \mathscr{T}$. Thus Theorem 3.1 is established.

The steps of the above proof may be analyzed as follows:

1. Produce a functional equation (namely (3.4) for $f_{\mathscr{R}}(x)$-a linear, second order $q$-difference equation).

2. Produce some $q$-series representation of the essentially unique solution of the functional equation.

3. Deduce the theorem from some $q$-series and product identity like (3.6).

This analysis applies to the vast majority of results that have been obtained in this area (see $\S \S 1,4$, and 5 of PI for examples of this technique).

The following two questions then naturally arise when one looks to how widely the above technique may be applied.

Question 1. For what partition ideals $C$ does

$$
f_{C}(x ; q)=\sum_{\left\{f_{i}\right\} \in C} x^{\Sigma f_{i}} q^{\Sigma f_{i} \cdot i}
$$

satisfy a finite linear $q$-difference equation with polynomial coefficients?

Question 2. What finite linear $q$-difference equations with polynomial coefficients (such as (3.4)) have solutions that can be represented by "higher-dimensional" $q$-series?

Question 2 leaves (intentionally) vague the concept of $q$-series. What one wants are solutions that are as amenable to manipulation and identity proving as the basic hypergeometric series studied by Bailey [16] and Slater [37]:

$$
\sum_{n \geqq 0} \frac{\left(a_{1}\right)_{n} \cdots\left(a_{r}\right)_{n} t^{n}}{(q)_{n}\left(b_{1}\right)_{n} \cdots\left(b_{s}\right)_{r}},
$$

where $(A)_{n}=(1-A)(1-A q) \cdots\left(1-A q^{n-1}\right)$. As we shall see in $\S 6$, presumably acceptable solutions would involve many-fold $q$-series, i.e. higherdimensional $q$-series and limiting cases thereof. F. H. Jackson's basic 
Appell series [28] (see also [10]) provide examples of two-dimensional $q$-series.

4. Linked partition ideals. The dissection of $\mathscr{R}$ into $\mathscr{R}_{1} \cup \mathscr{R}_{2}$ utilized in $\S 3$ to prove that $f_{\mathscr{R}}(x)$ satisfies (3.4) may be extended to a wide class of partition ideals. We shall now present a series of new concepts which will allow us to define a linked partition ideal. We shall see in Theorem 4.1 that for each linked partition ideal $C, f_{C}(x ; q)$ satisfies a finite $q$-difference equation with polynomial coefficients.

Definition 5. If $C$ is a partition ideal,

$$
C^{(j)}=\left\{\left\{f_{i}\right\} \in C \mid f_{1}=f_{2}=\cdots=f_{j}=0\right\} .
$$

Definition 6. We denote by $\varphi$ the bijection $\mathscr{S}_{\rightarrow} \mathscr{S}^{(1)}$ given by

$$
\begin{aligned}
& \varphi\left(\left\{f_{i}\right\}\right)=\left\{g_{i}\right\} \quad \text { where } g_{i}=0 \quad \text { if } i=1, \\
& =f_{i-1} \text { if } i>1 \text {. }
\end{aligned}
$$

In intuitive terms, $\varphi$ adds one to each part of each partition since

$$
\sigma\left(\varphi\left(\left\{f_{i}\right\}\right)\right)=\sum_{i=2}^{\infty} f_{i-1} \cdot i=\sum_{i=1}^{\infty} f_{i}(i+1) .
$$

Definition 7. We say a partition ideal $C$ has a modulus $m$ if $m$ is an integer such that $\varphi^{m} C=C^{(m)}$.

If we look back at $\S 3$, we see immediately that the least modulus for $\mathscr{R}$ is 1 and $\mathscr{T}$ has least modulus 5 .

Definition 8. If $C$ has a modulus, say $m$, and if the set

$$
L_{C}=\left\{\left\{f_{i}\right\} \in C \mid f_{i}=0 \text { for } i>m\right\}
$$

is finite, then we say that $C$ is a locally finite partition ideal.

Thus $L_{\mathscr{R}}$ is the two element set consisting of $\mathbf{0}=\{0,0,0, \cdots\}$ and $\{1,0,0,0, \cdots\}$, while $L_{\mathscr{T}}$ is infinite and consists of all elements of $\mathscr{S}$ of the form $\left\{m_{1}, 0,0, m_{2}, 0,0,0,0,0\right\}$, where $m_{1} \geqq 0, m_{2} \geqq 0$.

It is clear that if $C$ has a modulus, then for $C$ to be locally finite it is necessary and sufficient that there exists an absolute constant $M$ such that $f_{i} \leqq M$ for all $i$ whenever $\left\{f_{i}\right\} \in C$.

It is also clear that if $m$ is the least positive modulus for $C$, then every modulus $M$ is a multiple of $m$. This is because if $M=k m+r, 0 \leqq r<m$, then

$$
C=\varphi^{-M} C^{(M)}=\varphi^{-r} \varphi^{-k m} C^{(M)}=\varphi^{-r} C^{(M-k m)}=\varphi^{-r} C^{(r)} ;
$$

hence $r=0$, otherwise the minimality of $m$ is contradicted. Generally when we refer to the modulus of $C$, we shall mean the least positive modulus unless otherwise indicated. 
Definition 9. For each $\pi=\left\{f_{i}\right\} \in \mathscr{S}, \pi_{<N}=\left\{f_{i<N}\right\}$ shall denote $\left\{f_{1}, f_{2}, f_{3}, \cdots, f_{N-1}, 0,0,0, \cdots\right\}$, while $\pi_{\leqq N}=\left\{f_{i \leqq N}\right\}$ denotes $\left\{f_{1}, f_{2}\right.$, $\left.f_{3}, \cdots, f_{N}, 0,0,0, \cdots\right\}$.

DEFINITION 10. We write $\left\{f_{i}\right\} \oplus\left\{g_{i}\right\}=\left\{f_{i}+g_{i}\right\}$.

Definition 11. Suppose $C$ is locally finite of modulus $m$. We shall say that $\pi=\left\{f_{i}\right\} \in L_{C}$ is linked with span $l=l(\pi)$ if (1) $l$ is the least positive integer such that $\left\{f_{i}\right\} \oplus \varphi^{l m}\left\{g_{i}\right\} \in C$ for some $\left\{g_{i}\right\} \in L_{C}$ with $\left\{g_{i}\right\} \neq \mathbf{0}$, and (2) $\left\{f_{i}\right\} \oplus\left\{h_{i}\right\} \in C$ whenever $\left\{h_{i}\right\} \in C^{(l m)}$ and $\left\{f_{i}\right\} \oplus\left\{h_{i \leqq(l+1) m}\right\} \in C$. We say $C$ is linked if $C$ is locally finite and every element of $L_{C}$ is linked.

Definition 12. Suppose $C$ is linked. For each $\pi \in L_{C}$, we define a subset $\mathscr{L}_{C}(\pi)$ of $L_{C}$ called the linking set for $\pi$ as those $\pi^{\prime} \in L_{C}$ such that $\pi \oplus \varphi^{l(\pi) \cdot m} \pi^{\prime} \in C$.

Returning to $\mathscr{R}$ we see that $\mathscr{R}$ is linked since $\mathbf{0}$ has span 1 and $\mathscr{L}_{\mathscr{R}}(\mathbf{0})=$ $L_{\mathscr{R}}$ while $i=\{1,0,0,0, \cdots\}$ has span 2 and $\mathscr{L}_{\mathscr{R}}(i)=L_{\mathscr{R}}$ also.

THEOREM 4.1. If $C$ is a linked partition ideal, then $f_{C}(x ; q)$ satisfies $a$ finite homogeneous linear $q$-difference equation whose coefficients are polynomials in $x$ and $q$.

Proof. We begin by deriving several results about $C$ from the fact that $C$ is linked. First we know that $C$ has at least one modulus, say $m$, for which we also know that the corresponding $L_{C}$ is finite. Let $\kappa$ denote the cardinality of $L_{C}$, and we write $L_{C}=\left\{\pi_{0}, \pi_{1}, \pi_{2}, \cdots, \pi_{\kappa-1}\right\}$, where $\pi_{0}=\mathbf{0}$.

Next we define for each $\pi_{i} \in L_{C}$

$$
H_{i}(x)=\sum_{\pi^{\prime}}^{\dagger} x^{\#\left(\pi^{\prime}\right)} q^{\sigma\left(\pi^{\prime}\right)}
$$

where $\sum^{\dagger}$ runs over all $\pi^{\prime}=\left\{f_{i}\right\} \in C$ such that $\left\{f_{j \leqq m}\right\}=\pi_{i}$. We may now easily deduce several important relations for these functions. First

$$
H_{0}(x)=\sum_{\pi \in C^{(m)}} x^{\#(\pi)} q^{\sigma(\pi)}=\sum_{\pi \in C} x^{\#(\pi)} q^{\sigma(\pi)+m \cdot \#(\pi)}=f_{C}\left(x q^{m} ; q\right) .
$$

Next for each $\pi_{i} \in L_{C}$,

$$
\begin{aligned}
& H_{i}(x)=\sum_{\substack{\pi=\pi_{i} \oplus \varphi^{l}\left(\pi_{i}\right) m_{\pi^{*}} \\
\pi_{\cong m}^{*} \in \mathscr{L}_{\sigma\left(\pi_{i}\right) ; \pi^{*} \in C}}} x^{\#(\pi)} q^{\sigma(\pi)} \\
& =x^{\#\left(\pi_{\imath}\right)} q^{\sigma\left(\pi_{\imath}\right)} \sum_{\substack{\pi=\varphi^{2\left(\pi_{i}\right) m} \pi^{*} \\
\pi^{*}}} x^{\#(\pi)} q^{\sigma(\pi)} \\
& =x^{\#\left(\pi_{i}\right)} q^{\sigma\left(\pi_{\imath}\right)} \sum_{\pi^{*} \leqq m \in \mathscr{L}_{C\left(\pi_{i}\right) ; \pi^{*} \in C}} x^{\#\left(\pi^{*}\right)} q^{\sigma\left(\pi^{*}\right)+l\left(\pi_{\imath}\right) m \#\left(\pi^{*}\right)} \\
& =x^{\#\left(\pi_{i}\right)} q^{\sigma\left(\pi_{i}\right)} \sum_{\pi_{j} \in \mathscr{L}_{o\left(\pi_{i}\right)}} H_{j}\left(x q^{l\left(\pi_{\imath}\right) m}\right) .
\end{aligned}
$$


Now the (4.3) $)_{\mathrm{i}}$ constitute a system of $\kappa q$-difference equations in the $\kappa$ functions $H_{i}(x)$. All that remains (by (4.2)) is to show that this system may be reduced to a single $q$-difference equation for $H_{0}(x)$.

Here we shall rely on the anology with linear differential equations. In [30, Chapter 5, §5], Murray and Miller provide an algorithm for reducing a system of first order differential equations in $y_{1}, \cdots, y_{n}$ to $n$ higher order equations wherein the $j$ th equation involves only $y_{j}$. For our purposes the significant result lies in the first part of their Theorem 7 [30, p. 129]:

MURRAY-Miller TheORem. Every function $y$ which is a component of the solution of

$$
\dot{y}_{j}=\sum_{k=1}^{n} p_{j k}(x) y_{k}+q_{j}(x), \quad j=1, \cdots, n,
$$

is a solution of a single linear differential equation of order $r$ where $r \leqq n$.

On examination of the algorithm that is used to prove this theorem, we see that the differentiation operator may be replaced by any linear operator $L$ on the field of functions analytic in some complex domain $\mathscr{D}$ subject to the restriction

$$
L(a(x) y)=\alpha_{L}(x) y+\beta_{L}(x) L(y),
$$

where $\alpha_{L}(x), \beta_{L}(x)$ and $a(x)$ all lie in the field of functions that contains the coefficients of the equations considered, and where $\beta_{L}(x) \equiv 0$ if and only if $a(x) \equiv 0$.

When $L=d \mid d x, \alpha_{L}(x)=a^{\prime}(x)$ and $\beta_{L}(x)=a(x)$. When $L(f(x))=f(x q)$, then $\alpha_{L}(x)=0$ and $\beta_{l}(x)=a(x q)$.

Modified Murray-Miller Theorem. Let $\mathscr{F}=C(x)$ be the field of rational functions of the complex variable $x$. Let $L$ be a linear operator subject to the above described conditions. Every function $y$ which is part of a solution of

$$
L\left(y_{j}\right)=\sum_{k=1}^{n} p_{j k}(x) y_{k}+q_{j}(x), \quad 1 \leqq j \leqq n, \quad p_{j k}(x) \in \mathscr{F}, \quad q_{j}(x) \in \mathscr{F} .
$$

satisfies an equation of the form

$$
\sum_{h=0}^{r} s_{h}(x) L^{h}(y)=t(x), \quad \text { where } r \leqq n, \quad s_{h}(x) \in \mathscr{F}, \quad t(x) \in \mathscr{F} .
$$

Furthermore if the $q_{j}(x)$ are all zero so are the $t(x)$. 
The final assertion in this theorem is easily deduced by examination of the algorithm used in the proof.

For our purposes $L$ will denote the operator given by $L(f(x))=f\left(x q^{m}\right)$, $0<|q|<1$. We must now transform the system (4.3) into the form (4.4). First we write

$$
h_{i j}(x)=H_{i}\left(x^{-1} q^{-j m}\right), \quad 0 \leqq i \leqq \kappa-1, \quad 0 \leqq j<\omega_{i},
$$

where $\omega_{i}$ is the largest value of $\omega$ such that $H_{i}\left(x q^{\omega m}\right)$ actually appears in the system (4.3) $)_{\mathrm{i}}$ (by $(4.3)_{0}$ we see that $\omega_{i} \geqq 1$ always). The system $(4.3)_{\mathrm{i}}$ is now equivalent to the following system of $\Omega=\omega_{0}+\omega_{1}+\cdots+\omega_{\kappa-1}$ equations in $\Omega$ dependent variables $h_{i j}$.

$$
\begin{aligned}
L\left(h_{i, \omega_{2}-1}\right) & =h_{i, \omega_{2}-1}\left(x q^{m}\right)=\sum_{j=0}^{\kappa} \sum_{k=0}^{\omega_{2}-1} p_{j, k}(i ; x) h_{j, k}(x), \quad 0 \leqq i \leqq \kappa-1, \\
L\left(h_{i j}\right) & =h_{i, j+1}, \quad 0 \leqq i \leqq \kappa-1, \quad 0 \leqq j<\omega_{i}-1,
\end{aligned}
$$

where the top system of equations in (4.5) is merely the (4.3) with $x$ replaced by $x^{-1} q^{-\omega_{\imath} m}$. Since (4.5) is of the form (4.4), we see that Theorem 4.1 follows. Furthermore the order of the implied $q$-difference equation is $\leqq m \Omega$.

To illustrate the workings of Theorem 4.1, let us return to $f_{\mathscr{R}}(x ; q)$. In this case $(4.3)_{0}$ becomes

and (4.3) $)_{1}$ becomes

$$
H_{0}(x)=H_{0}(x q)+H_{1}(x q),
$$

$$
H_{1}(x)=x q\left(H_{0}\left(x q^{2}\right)+H_{1}\left(x q^{2}\right)\right) .
$$

Hence eliminating $H_{1}$ from these equations, we find

or

$$
H_{0}(x)-H_{0}(x q)=x q^{2}\left(H_{0}\left(x q^{3}\right)+H_{0}\left(x q^{2}\right)-H_{0}\left(x q^{3}\right)\right),
$$

Hence

$$
H_{0}(x)=H_{0}(x q)+x q^{2} H_{0}\left(x q^{2}\right) .
$$

$$
f_{\mathscr{R}}(x ; q)=f_{\mathscr{R}}(x q ; q)+x q f_{\mathscr{R}}\left(x q^{2} ; q\right) \text {. }
$$

In [2], H. L. Alder defined polynomials $G_{k, n}(q)$ that have subsequently become known as the Alder polynomials. These polynomials may be defined by

$$
\sum_{\pi \in \mathcal{P}_{0, k, k}} x^{\#(\pi)} q^{\sigma(\pi)}=\sum_{n \geqq 0} G_{k, n}(q) x^{n} /(q)_{n},
$$

where $\mathscr{R}_{0, k, k}=\left\{\left\{f_{i}\right\} \in \mathscr{S} \mid f_{i}+f_{i+1} \leqq k-1\right\}$. (The truth of this assertion follows from equations (5.2) and (5.15) in the next section after comparison with Alder's original definition.)

The Alder polynomials have been found to be of number-theoretic 
significance in [13]. We shall now show that any linked partition ideal has an associated family of Alder polynomials:

THEOREM 4.2. Suppose that $C$ is a linked partition ideal with a modulus $m$. Then there exists a family of polynomials $A_{n}(C ; m ; q)$ (to be called the "Alder polynomials for $C$ relative to $m$ " or more briefly the C-Alder polynomials) such that

$$
f_{C}(x ; q)=\sum_{\pi \in C} x^{\#(\pi)} q^{\sigma(\pi)}=\sum_{n \geqq 0} \frac{A_{n}(C ; m, q) x^{n}}{\left(q^{m} ; q^{m}\right)_{n}} .
$$

Proof. It is clear that the $A_{n}(C, m ; q)$ exist as functions of $q$ since $f_{C}(x ; q)$ is analytic in $x$ around $x=0$ for $|q|<1$. The problem is to show that they are polynomials. Referring to the proof of Theorem 4.1, we define

$$
\begin{aligned}
& H_{j}(x)=\sum_{n \geqq 1} \frac{a_{n}(j ; q) x^{n}}{\left(q^{m} ; q^{m}\right)_{n-1}}, \quad 1 \leqq j \leqq \kappa-1, \\
& H_{0}(x)=\sum_{n \geqq 0} \frac{a_{n}(0 ; q) x^{n} q^{m n}}{\left(q^{m} ; q^{m}\right)_{n}}
\end{aligned}
$$

which is admissible since $a_{0}(0 ; q)=1, a_{0}(j ; q)=0$ for $1 \leqq j \leqq \kappa-1$, and $a_{n}(j ; q)=0$ for $n<0$.

Furthermore when $i=0$ in $(4.3)_{\mathrm{i}}$, we see that $\pi_{0}=0, \#\left(\pi_{0}\right)=0, \sigma\left(\pi_{0}\right)=0$, and every element of $L_{C}$ is linked to $\pi_{0}$ with span 1 . Hence (4.3) is just

$$
H_{0}(x)=\sum_{j=0}^{\kappa-1} H_{j}\left(x q^{m}\right)
$$

therefore for $n \geqq 1$,

or

$$
\frac{a_{n}(0 ; q) q^{m n}}{\left(q^{m} ; q^{m}\right)_{n}}=\frac{q^{2 m n} a_{n}(0 ; q)}{\left(q^{m} ; q^{m}\right)_{n}}+\sum_{j=1}^{\kappa-1} \frac{q^{m n} a_{n}(j ; q)}{\left(q^{m} ; q^{m}\right)_{n-1}},
$$

$$
a_{n}(0 ; q)=\sum_{j=1}^{\kappa-1} a_{n}(j ; q) .
$$

For $i>0$, we see from (4.3), that for $n>0$,

$$
\begin{aligned}
& \frac{a_{n}(i ; q)}{\left(q^{m} ; q^{m}\right)_{n-1}}=q^{\sigma\left(\pi_{2}\right)} \sum_{\pi_{j} \in \mathscr{L}_{Q}\left(\pi_{2}\right) ; \pi_{j} \neq \pi_{0}} q^{l\left(\pi_{i}\right) m\left(n-\#\left(\pi_{i}\right)\right)} \frac{a_{n-\#\left(\pi_{i}\right)}(j ; q)}{\left(q^{m} ; q^{m}\right)_{n-\#\left(\pi_{i}\right)-1}} \\
& +\frac{q^{\sigma\left(\pi_{i}\right)+\left(l\left(\pi_{i}\right)+1\right) m\left(n-\#\left(\pi_{i}\right)\right)} a_{n-\#\left(\pi_{i}\right)}(0 ; q)}{\left(q^{m} ; q^{m}\right)_{n-\#\left(\pi_{i}\right)}},
\end{aligned}
$$


or

$$
\begin{aligned}
& a_{n}(i ; q)=q^{\sigma\left(\pi_{i}\right)} \sum_{\pi_{j \in \mathscr{L}}\left(\pi_{i}\right) ; \pi_{j} \neq \pi_{0}} q^{l\left(\pi_{i}\right) m\left(n-\#\left(\pi_{i}\right)\right)} a_{n-\#\left(\pi_{\imath}\right)}(j ; q) \\
& \cdot \prod_{h=0}^{\#\left(\pi_{i}\right)-1}\left(1-q^{m\left(n-\#\left(\pi_{i}\right)+h\right)}\right) \\
& +q^{\sigma\left(\pi_{i}\right)+\left(l\left(\pi_{\imath}\right)+1\right) m\left(n-\#\left(\pi_{\imath}\right)\right)} a_{n-\#\left(\pi_{i}\right)}(0 ; q) \\
& \cdot \prod_{h=0}^{\#\left(\pi_{i}\right)}\left(1-q^{m\left(n-\#\left(\pi_{i}\right)+h\right)}\right) \text {. }
\end{aligned}
$$

It is now an easy matter to prove that the functions $a_{n}(j ; q)$ are all polynomials and are uniquely determined by (4.8), (4.9), and the initial conditions $a_{0}(0 ; q)=1, a_{0}(j ; q)=0$ for $j>0, a_{n}(j ; q)=0$ for $n<0$. The initial conditions establish our assertion for $n=0$. Assume the result for $n<n_{0}$. Then for $i \neq 0$, we know $\#\left(\pi_{i}\right)>0$; hence the fact that $a_{n_{0}}(i ; q)$ is unique and a polynomial follows from (4.9) (note that if $\#\left(\pi_{i}\right)>n_{0}$, then $\left.a_{n_{0}-\#\left(\pi_{i}\right)}(j ; q)=0\right)$. For $i=0$, we now use (4.8) to establish the result for $a_{n_{0}}(0 ; q)$ and, therefore, to complete the induction. To conclude we note that (by (4.2)),

$$
\sum_{n \geqq 0} \frac{A_{n}(C ; m ; q) x^{m} q^{m}}{\left(q^{m} ; q^{m}\right)_{n}}=f_{C}\left(x q^{m} ; q\right)=H_{0}(x)=\sum_{n \geqq 0} \frac{a_{n}(0 ; q) x^{m} q^{m}}{\left(q^{m} ; q^{m}\right)_{n}}
$$

hence $A_{n}(C ; m ; q)=a_{n}(0 ; q)$ is a polynomial in $q$.

COROLlaRY. The $H_{j}(x)$ are the unique functions analytic in $x$ at $x=0$ that satisfy the system $(4.3)_{\mathrm{i}}$ and the initial conditions $H_{0}(0)=1, H_{j}(x)=0$, $1 \leqq j \leqq \kappa-1$.

Proof. The uniqueness of the $a_{n}(j ; q)$ proved in Theorem 4.2 was derived using only the facts described in the Corollary.

5. Discussion of linked partition ideals. The linked partition ideals introduced in $\$ 4$ appear in almost all of the partition identities of the Rogers-Ramanujan type. As a vehicle for discussion of Theorem 4.1 we state the most general identity known that contains the Rogers-Ramanujan identities as a special case [14].

THEOREM 5.1. Let $0 \leqq \lambda \leqq a \leqq k$ be integers. If $\lambda$ is even, let $A_{\lambda, k, a}(n)$ denote the number of partitions of $n$ into parts such that no part $\not \equiv 0$ $(\bmod \lambda+1)$ may be repeated and no part is $\equiv 0$,

$$
\pm\left(a-\frac{1}{2} \lambda\right)(\lambda+1) \quad(\bmod (2 k-\lambda+1)(\lambda+1)) .
$$

If $\lambda$ is odd, let $A_{\lambda, k, a}(n)$ denote the number of partitions of $n$ into parts such that no part $\not \equiv 0\left(\bmod \frac{1}{2}(\lambda+1)\right)$ may be repeated, no part is $\equiv \lambda+1$ 
$(\bmod 2 \lambda+2)$, and no part is $\equiv 0, \pm(2 a-\lambda) \frac{1}{2}(\lambda+1)(\bmod (2 k-\lambda+1)(\lambda+1))$. Let $B_{\lambda, k, a}(n)$ denote the number of partitions of $n$ of the form $b_{1}+\cdots+b_{s}$, with $b_{i} \geqq b_{i+1}$, no parts $\not \equiv 0(\bmod \lambda+1)$ are repeated, $b_{i}-b_{i+k-1} \geqq \lambda+1$ with strict inequality if $(\lambda+1) \mid b_{i}$ and at most $a-1$ parts are $\leqq \lambda+1$. Then $A_{\lambda, k, a}(n)=B_{\lambda, k, a}(n)$ for each $n$.

Remarks. The above theorem may be easily phrased in terms of partition ideals. Namely, let

$$
\begin{gathered}
\mathscr{B}_{\lambda, k, a}=\left\{\left\{f_{i}\right\} \in \mathscr{S} \mid \sum_{j=0}^{\lambda} f_{m+j} \leqq k-1 \text { and } \sum_{j=0}^{\lambda+1} f_{m(\lambda+1)+j} \leqq k-1\right. \\
\text { for each } \left.m \geqq 1, f_{1}+\cdots+f_{\lambda+1} \leqq a-1, \text { and } f_{i}>1 \text { implies }(\lambda+1) \mid i\right\}, \\
\mathscr{A}_{2 \lambda, k, a}=\left\{\left\{f_{i}\right\} \in \mathscr{S} \mid f_{i}>1 \text { implies }(2 \lambda+1) \mid i ; \text { and } f_{i}>0\right. \text { implies } \\
i \not \equiv 0, \pm(a-\lambda)(2 \lambda+1)(\bmod (2 k-2 \lambda+1)(2 \lambda+1))\}, \\
\mathscr{A}_{2 \lambda+1, k, a}=\left\{\left\{f_{i}\right\} \in \mathscr{S} \mid f_{i}>1 \text { implies }(\lambda+1) \mid i ; f_{i}>0\right. \text { implies } \\
i \not \equiv 2 \lambda+2(\bmod 4 \lambda+4) \text { and } i \not \equiv 0, \\
\pm(2 a-2 \lambda-1)(\lambda+1)(\bmod (2 k-2 \lambda)(2 \lambda+2))\} .
\end{gathered}
$$

Then Theorem 5.1 is equivalent to $\mathscr{A}_{\lambda, k, a} \sim^{\mathrm{PT}} \mathscr{B}_{\lambda, k, a}$ provided $0 \leqq \lambda \leqq a \leqq k$.

The first Rogers-Ramanujan identity is the case $\lambda=0, k=a=2$. The second Rogers-Ramanujan identity is the case $\lambda=0, k=2, a=1$. Theorem 5.1 contains a number of other well-known partition identities as special cases; a weaker form of this theorem which stipulates $k \geqq 2 \lambda-1$ is discussed in $\S 1$ of PI and proved in [6].

It is easy to show that $\mathscr{B}_{\lambda, k, k}$ is a linked partition ideal with least modulus $\lambda+1$ and that $L_{\mathscr{B} \lambda, k, k}$ is a set consisting of

$$
\kappa=k+(k-1)\left(\begin{array}{l}
\lambda \\
1
\end{array}\right)+(k-2)\left(\begin{array}{l}
\lambda \\
2
\end{array}\right)+\cdots+(k-\lambda)\left(\begin{array}{l}
\lambda \\
\lambda
\end{array}\right)=2^{\lambda-1}(2 k-\lambda)
$$

elements. Furthermore the span of each element is at most 2 so that in terms of Theorem 4.1 the order of the resulting $q$-difference equation is at most $(\lambda+1) \cdot 2 \cdot 2^{\lambda-1}(2 k-\lambda)=(\lambda+1) 2^{\lambda}(2 k-\lambda)$. In order to prove Theorem 5.1, much more subtlety is required than is available from a direct application of Theorem 4.1, and in fact a system of $q$-difference equations is obtained from which it is possible to deduce a single one of order $k(\lambda+1)$ for $f_{\mathscr{B}_{\lambda, k}, k}(x ; q)$. The technique of proof relies on cutting the number of auxiliary functions down from $\kappa=2^{\lambda-1}(2 k-\lambda)$ to $2 k+1$. In fact, in most interesting special cases, the relevant $q$-difference equation will have a much lower order than the upper bound given in Theorem 4.1. Below we outline the general proof of Theorem 5.1. The details of this work will appear in [14]. 
Sketch of Proof of Theorem 5.1. We begin with functions that correspond to $\phi(x)$ defined in equation (3.5):

$$
\begin{aligned}
\varphi_{\lambda, k, i}(x)= & \frac{(-x q)_{\infty}}{\left(x^{2} q^{2 \lambda+2} ; q^{2 \lambda+2}\right)_{\infty}} \sum_{n=0}^{\infty}(-1)^{n} x^{k n} q^{(\lambda+1)\left((2 k-\lambda+1) n^{2}+(2 k-2 i+1) n\right) / 2} \\
& \cdot \frac{\left(1-x^{i} q^{(2 n+1) i(\lambda+1)}\right)\left(x^{2} q^{2 \lambda+2} ; q^{2 \lambda+2}\right)_{n}(-q)_{\lambda n+n}}{\left(q^{2 \lambda+2} ; q^{2 \lambda+2}\right)_{n}(-x q)_{\lambda n+\lambda+n}} ; \\
\psi_{\lambda, k, i}(x)= & \frac{(-x q)_{\infty}}{\left(x^{2} q^{2 \lambda+2} ; q^{2 \lambda+2}\right)_{\infty}} \sum_{n=0}^{\infty}(-1)^{n} x^{k n} q^{(\lambda+1)\left((2 k-\lambda+1) n^{2}+(2 k-2 i+1) n\right) / 2} \\
& \cdot \frac{\left(x^{2} q^{2 \lambda+2} ; q^{2 \lambda+2}\right)_{n}(-q)_{(\lambda+1) n}}{\left(q^{2 \lambda+2} ; q^{2 \lambda+2}\right)_{n}(-x q)_{\lambda n+n}} \\
& \times\left(1-\frac{x^{i} q^{(\lambda+1)(2 n+1)(2 i-\lambda) / 2}\left(-q^{\lambda n+n+1}\right)_{\lambda}}{\left(-x q^{n \lambda+n+1}\right)_{\lambda}}\right)
\end{aligned}
$$

where $(a ; q)_{n}=(a)_{n}=(1-a)(1-a q) \cdots\left(1-a q^{n-1}\right)$, and $(a ; q)_{\infty}=(a)_{\infty}=$ $\lim _{n \rightarrow \infty}(a ; q)_{n}$.

Note that $\varphi_{0,2,2}(x)=\psi_{0,2,2}(x)=\varphi(x)$.

It is possible to prove that these functions satisfy the following relations:

$$
\begin{gathered}
\varphi_{\lambda, k, i}(x)-\varphi_{\lambda, k, i-1}(x)=\left(x q^{\lambda+1}\right)^{i-1} \psi_{\lambda, k, k-i+1}\left(x q^{\lambda+1}\right) ; \\
\psi_{\lambda, k, i}(x)=\sum_{j=0}^{\lambda} x^{j} q^{\jmath(j+1) / 2}\left[\begin{array}{l}
\lambda \\
j
\end{array}\right] \varphi_{\lambda, k, i-j}(x) ; \\
\varphi_{\lambda, k,-i}(x)=-\left(x q^{\lambda+1}\right)^{-i} \varphi_{\lambda, k, i}(x),
\end{gathered}
$$

where

$$
\begin{aligned}
{\left[\begin{array}{l}
\lambda \\
j
\end{array}\right] } & =\frac{(q)_{\lambda}}{(q)_{j}(q)_{\lambda-j}}, & & 0 \leqq j \leqq \lambda, \\
& =0, & & \text { otherwise. }
\end{aligned}
$$

Utilizing Jacobi's triple product identity [7, pp. 169-170], one may easily deduce from (5.2) that

$$
\begin{aligned}
\psi_{\lambda, k, i}(1)= & (-q)_{\infty}\left(q^{2 \lambda+2} ; q^{2 \lambda+2}\right)_{\infty}^{-1}\left(q^{(i-\lambda / 2)(\lambda+1)} ; q^{(2 k-\lambda+1)(\lambda+1)}\right)_{\infty} \\
& \cdot\left(q^{(2 k-\lambda / 2-i+1)(\lambda+1)} ; q^{(2 k-\lambda+1)(\lambda+1)}\right)_{\infty}\left(q^{(2 k-\lambda+1)(\lambda+1)} ; q^{(2 k-\lambda+1)(\lambda+1)}\right)_{\infty} \\
(5.7)= & \sum_{n=0}^{\infty} A_{\lambda, k, a}(n) q^{n} .
\end{aligned}
$$

On the other hand, we may define generating functions related to the partition ideals $\mathscr{B}_{\lambda, k, a}$. Namely

$$
j_{\lambda, k, a}(x)=\sum_{\left\{f_{\imath}\right\} \in \mathscr{B}_{\lambda, k, a}} x^{\#\left(\left\{f_{\imath}\right\}\right)} q^{\sigma\left(\left\{f_{\imath}\right\}\right)},
$$


and

$$
h_{\lambda, j, a}(x)=\sum_{\left\{f_{i}\right\} \in \mathscr{B}_{\lambda, k, a}} x^{\#\left(\left\{f_{i}\right\}\right)} q^{\sigma\left(\left\{f_{i}\right\}\right)} \quad\left(\text { where }\left\{f_{i \leqq \lambda}\right\}=\mathbf{0}\right) .
$$

It is a straightforward matter to prove that

$$
h_{\lambda, k, a}(x)-h_{\lambda, k, a-1}(x)=\left(x q^{\lambda+1}\right)^{a-1} j_{\lambda, k, k-a+1}\left(x q^{\lambda+1}\right),
$$

a perfect replica of (5.3).

Surprisingly instead of a replica of (5.4), the following relation holds.

$$
\begin{aligned}
j_{\lambda, k, a}(x)= & \sum_{j=0}^{a} x^{j} q^{j(j+1) / 2}\left[\begin{array}{l}
\lambda \\
j
\end{array}\right] h_{\lambda, k, a-j}(x) \\
& +\sum_{l \geqq 2}(-1)^{l-1} \sum_{r=0}^{k} g(l ; k, \lambda, r, a ; x ; q) h_{\lambda, k, r}\left(x q^{(l-1)(\lambda+1)}\right),
\end{aligned}
$$

where

$$
\begin{aligned}
g(l ; k, \lambda, & r, a ; x ; q) \\
= & \sum_{\mathscr{C}} x^{k(l-1)+\Sigma A-\Sigma D} q^{\Sigma_{b=1}^{l-1}\left(k-D_{b}+A_{b}\right) b(\lambda+1)+D_{b}\left(D_{b}+1\right) / 2} \\
& \cdot q^{(\Sigma A-\Sigma D)(\Sigma A-\Sigma D+1) / 2}\left[\begin{array}{c}
\lambda \\
D_{1}
\end{array}\right]\left[\begin{array}{c}
\lambda \\
D_{2}
\end{array}\right] \cdots\left[\begin{array}{c}
\lambda \\
D_{l-1}
\end{array}\right]\left[\begin{array}{c}
\lambda \\
\Sigma A-\Sigma D
\end{array}\right],
\end{aligned}
$$

with $\Sigma A=A_{0}+A_{1}+\cdots+A_{l+1}, \Sigma D=D_{1}+\cdots+D_{l-1}$, and $\mathscr{C}$ is the set of those (2l-1)-tuples $\left(A_{0}, A_{1}, \cdots, A_{l-1}, D_{1}, \cdots, D_{l-1}\right)$ that satisfy $A_{0}+k-D_{1} \leqq i-1, \quad A_{l-1}=D_{l-1}-r, \quad 0 \leqq A_{0} \leqq \lambda, \quad 1 \leqq D_{j} \leqq k, \quad 0 \leqq A_{j} \leqq D_{j}$, $A_{j} \leqq D_{j}+D_{j+1}-k-1$.

From these $q$-difference equations (5.8)-(5.11), it is possible to deduce that

$$
p_{\lambda, k, i}(x)=h_{\lambda, k, i}(x)+\sum_{l \geqq 2}(-1)^{l-1} \sum_{r=1}^{k} h_{\lambda, k, r}\left(x q^{(l-1)(\lambda+1)}\right) \gamma(l ; k, \lambda, r, i ; x ; q),
$$

with

$$
\begin{aligned}
& \gamma(l ; k, \lambda, r, i ; x ; q) \\
& \quad=x^{(l-1) k} \sum_{\mathscr{D}} q^{\Sigma_{b=1}^{l-1}\left(k-D_{b}+A_{b}\right) b(\lambda+1)+D_{b}\left(D_{b}+1\right) / 2}\left[\begin{array}{c}
\lambda \\
D_{1}
\end{array}\right]\left[\begin{array}{c}
\lambda \\
D_{2}
\end{array}\right] \cdots\left[\begin{array}{c}
\lambda \\
D_{l-1}
\end{array}\right],
\end{aligned}
$$

where $\mathscr{D}$ is the set of those $(2 l-2)$-tuples $\left(A_{1}, \cdots, A_{l-1}, D_{1}, \cdots, D_{l-1}\right)$ that satisfy $D_{2}+\cdots+D_{l-2}-A_{1}-\cdots-A_{l-2} \leqq i-k-r-1,1 \leqq D_{j} \leqq k, A_{l-1}=$ $D_{l-1}-r, 0 \leqq A_{j} \leqq D_{j}, A_{j} \leqq D_{j}+D_{j+1}-k-1$ (when $l=2, \mathscr{D}$ is to be defined by $\left.-D_{1} \leqq i-k-r-1, A_{1}=D_{1}-r\right)$. It turns out that

$$
\gamma(l ; k, \lambda, r, i ; x ; q) \equiv 0 \text { for } 0 \leqq i \leqq k-\lambda+1
$$


Hence for $\lambda \leqq a \leqq k$,

$$
\begin{aligned}
j_{\lambda, k, a}(x) & =x^{-a+1}\left(h_{\lambda, k, k-a+1}\left(x q^{-\lambda-1}\right)-h_{\lambda, k, k-a}\left(x q^{-\lambda-1}\right)\right) \\
& =x^{-a+1}\left(\varphi_{\lambda, k, k-a+1}\left(x q^{-\lambda-1}\right)-\varphi_{\lambda, k, k-a}\left(x q^{-\lambda-1}\right)\right) \\
& =\psi_{\lambda, k, a}(x) .
\end{aligned}
$$

Consequently,

$$
\begin{aligned}
\sum_{n \geqq 0} A_{\lambda, k, a}(n) q^{n} & =j_{\lambda, k, a}(1)=\psi_{\lambda, k, a}(1) \\
& =\sum_{\left\{f_{i}\right\} \in \mathscr{B} \lambda, k, a} q^{\sigma\left(\left\{f_{i}\right\}\right)}=\sum_{n \geqq 0} B_{\lambda, k, a}(n) q^{n} .
\end{aligned}
$$

Comparing coefficients of $q^{n}$ in the extremes of (5.16), we find that $A_{\lambda, k, a}(n)=B_{\lambda, k, a}(n)$ for each $n$, provided $\lambda \leqq a \leqq n$.

This concludes our sketch of the proof of Theorem 5.1.

The above work should make clear that the establishment of Theorem 5.1 relies on a lot of detailed results other than those implied by Theorem 4.1. In particular the polynomials $g(l ; k, \lambda, r, i ; x ; q)$ and $\gamma(l ; k, \lambda, r, i ; x ; q)$ undergo extensive consideration in [14] before one can represent them as sums of products of Gaussian polynomials $\left[\begin{array}{l}\lambda \\ D_{j}\end{array}\right]$.

Thus while Theorem 4.1 tells us that all linked partition ideals are amenable to attack via $q$-difference equations, it tells us nothing about what sorts of analytic representations (like $\varphi_{\lambda, k, a}(x)$ and $\psi_{\lambda, k, a}(x)$ ) are needed in order to establish new partition identities.

Before closing our discussion of Theorem 5.1, we remark that in [14] the result is generalized to include parameters satisfying $\lambda / 2<a \leqq k$, $\lambda \leqq k$; in fact this generalization appears in [6] subject to the extra condition $k \geqq 2 \lambda-1$. Also the following conjecture is raised in [14].

CONJECTURE. $A_{4,3,3}(n)=B_{4,3,3}^{0}(n)=p\left(\mathscr{B}^{0} ; n\right)$, where

$$
\begin{array}{r}
\mathscr{B}^{0}=\left\{\left\{f_{i}\right\} \in \mathscr{S} \mid f_{j}+f_{j+1}+\cdots+f_{j+4} \leqq 2, f_{5 j}+f_{5 j+1}+\cdots+f_{5 j+5} \leqq 2,\right. \\
f_{5 j-3}+f_{5 j-2} \leqq 1, f_{5 j-1}+f_{5 j+1} \leqq 1, f_{5 j-1}+f_{5 j}+f_{5 j+5}+f_{5 j+6} \leqq 3 \\
\text { for } \left.j \geqq 1, \text { and } f_{m}>1 \text { implies } 5 \mid m\right\} .
\end{array}
$$

This conjecture has been verified for $n \leqq 59$ where $A_{4,3,3}(59)=B_{4,3,3}^{0}(59)=$ 2938.

This problem seems to lie beyond the scope of the techniques introduced in [14], and if it is true, its proof should provide valuable insights and techniques for the theory of partition identities.

6. Representations of solutions of $q$-difference equations. Now we turn to Question 2 (stated at the end of §3), an aspect of this study where relatively little is known, and no truly general theorems like Theorem 4.1 are available for guidance. 
We begin by remarking that if we expand the $F(x)$ defined by (3.3) and (3.4) into a power series in $x$, say $\Sigma A_{n} x^{n}$, we find directly that $A_{0}=1$ and $\left(1-q^{n}\right) A_{n}=q^{2 n-1} A_{n-1}$. Hence

$$
F(x)=\sum_{n \geqq 0} \frac{q^{n^{2}} x^{n}}{(q)_{n}} .
$$

Comparing (6.1) with (3.5), we obtain an analytic identity:

$$
\sum_{n \geqq 0} \frac{q^{n^{2}} x^{n}}{(q)_{n}}=\sum_{n \geqq 0} \frac{(-1)^{n} x^{2 n} q^{n(5 n+1) / 2}\left(1-x^{2} q^{4 n+2}\right)}{(q)_{n}\left(x q^{n+1}\right)_{\infty}} .
$$

Thus two very different $q$-series representations of the solution of (3.4) are available to us.

In the same vein, one can prove that

$$
\begin{aligned}
& \varphi_{0,3,3}(x)=\sum_{m \geqq 0} \frac{x^{2 m} q^{2 m^{2}}\left(-x q^{2 m+1}\right)_{\infty}}{\left(q^{2} ; q^{2}\right)_{m}} \quad \text { [3, Theorem 8, p. 433] } \\
& \varphi_{0,4,4}(x)=\sum_{m \geqq 0} \frac{x^{3 m} q^{3 m^{2}}\left(x^{3} q^{6 m+3} ; q^{3}\right)_{\infty}}{\left(q^{3} ; q^{3}\right)_{m}\left(x q^{3 m+1}\right)_{\infty}} \quad \text { [3, Theorem 9, p. 444], } \\
& \varphi_{0,5,5}(x)=\sum_{j \geqq 0} \sum_{n \geqq 0} \frac{q^{4 n^{2}+12 n j+8 j^{2}+j}(-1)^{j}\left(x q^{4 n+2 j+1}\right)_{\infty} x^{4 n+6 j}}{\left(q^{4} ; q^{4}\right)_{n}\left(q^{2} ; q^{2}\right)_{j}\left(x^{4} q^{8 n+8 j+4} ; q^{4}\right)_{\infty}}
\end{aligned}
$$

Other series representations of $\varphi_{\lambda, k, i}(x)$ and $\psi_{\lambda, k, i}(x)$ have been found; however in all cases $(2 k-\lambda+1)(\lambda+1) \leqq 64$, and in these cases the only prime factors of $(2 k-\lambda+1)(\lambda+1)$ are 2, 3, 5, 7 (apart from the case $\lambda=0,2 k+1=11$ mentioned above and treated in [15]). These results are all (with the exception of [15]) to be found in the work of W. N. Bailey [16], [17], [18] and L. J. Slater [35], [36], [37]. To date the methods employed seem of limited scope as Slater's cataloging of known results includes only 130 identities.

Unfortunately most of the classical investigations of $q$-difference equations were concerned with existence theorems given specified analyticity conditions on the coefficients [1], [20], [21], [33]. We, however, have polynomial coefficients and already know that a unique solution exists given our simple boundary conditions. Our concern is not with the existence of a solution but with an appropriate representation of the solution.

Presumably the work of W. Hahn [25], [26], and F. Ryde [33], as well as the extension of Bailey's techniques contained in [15] may point the way to progress in this area, but much remains to be done.

7. Conclusion. I hope that this paper has given some indication of what has been done and what remains to be done in the theory of identities of the Rogers-Ramanujan type. 
Obviously a project that holds promise of rather immediate positive results is that of examining linked partition ideals $C$ with modulus $\leqq M_{1}$, all spans $\leqq M_{2}$ and $\left|L_{C}\right| \leqq M_{3}$. Clearly the number of such $C$ is finite, and this systematic approach promises to reveal $C$ with related $q$-difference equations of reasonably low order.

More important for the general goals, one hopes to make advances on the analytic questions considered in $\$ 6$.

Before concluding, we should point out that not all of the known theory of partition identities falls into the theory of linked partition ideals.

In particular, the theorems in [4] are not related to linked partition ideals except in the special case of Schur's theorem [34]. However examination of these results and comparison with the results in [5] suggest that a "dual" theory could be developed wherein $q$-recurrent sequences would replace $q$-difference equations.

There have been, however, other theorems developed that are definitely partition identities but do not at all concern an equivalence of partition ideals.

Frobenius [23, p. 523] introduced a notation for partitions that is especially useful here. He writes a partition $\pi$ as

$$
\left(\begin{array}{l}
a_{1}, a_{2}, \cdots, a_{r} \\
b_{1}, b_{2}, \cdots, b_{r}
\end{array}\right),
$$

where $\pi$ has Durfee square of side $r$ (see [27, p. 281] for discussion of the Durfee square), and where there are $a_{i}$ nodes to the right of the main diagonal in the $i$ th row of the Ferrars graph representation of $\pi$ and $b_{i}$ nodes below the main diagonal in the $i$ th column of the Ferrars graph representation of $\pi$. For example, if $\pi$ is $5+5+4+4+2+1+1$ (in $\mathscr{S}\{2,1,0,2,2,0,0,0, \cdots\})$, then the Ferrars graph is

and the Frobenius representation is

$$
\left(\begin{array}{l}
4,3,1,0 \\
6,3,1,0
\end{array}\right)
$$


We now define $F_{\max }(\pi)$ and $F_{\min }(\pi)$ to be $\max \left(a_{i}-b_{i}\right)$ and $\min \left(a_{i}-b_{i}\right)$, respectively. The following theorem then holds $[11],[12]$.

THEOREM 7.1. Let $Q_{k, a}(n)$ denote the number of partitions $\pi$ of $n$ such that $-a+2 \leqq F_{\min }(\pi) \leqq F_{\max }(\pi) \leqq 2 k-a-1$. Then $Q_{k, a}(n)=A_{0, k, a}(n)$ for all $n$.

It is not difficult to show that the Rogers-Ramanujan identities are equivalent to this result in the cases $k=2, a=2,1$. However all aspects of the multiple result

$$
Q_{k, a}(n)=A_{0, k, a}(n)=B_{0, k, a}(n)
$$

(implied by Theorem 5.1 ) are mostly unexplored for $k \geqq 3$. One could introduce a new lattice structure on partitions by the partial ordering $\pi_{1} \leqq \pi_{2}$ if all the columns in the Frobenius representation of $\pi_{1}$ appear in the Frobenius representation of $\pi_{2}$. Where such an approach would lead concerning partition identities has not been investigated at all.

Still other partition functions $H_{k, a}(n)$ have been found [13] such that $H_{k, a}(n)=A_{0, k, a}(n)$; here a study is made of the Alder polynomials $G_{k, a}(n ; q)$ defined by

$$
\varphi_{0, k, a}(x)=\sum_{n \geqq 0} \frac{G_{k, a}(n ; q) x^{n}}{(q)_{n}} .
$$

Finally, we mention that W. Connor [22] has obtained infinite families of partition identities also unlike any of the previously mentioned results. Connor's most important theorem in the simplest instance is a new set of partition-identities related to two elegant analytic theorems originally due to L. J. Rogers [32] but put in their most interesting form by Connor:

$$
\begin{gathered}
\sum_{n=0}^{\infty} \frac{q^{n^{2}+n}}{(q)_{2 n}}=\prod_{n=1 ; n \neq \pm 1, \pm 8, \pm 9,10(\bmod 20)}^{\infty}\left(1-q^{n}\right)^{-1} ; \\
\sum_{n=0}^{\infty} \frac{q^{n^{2}+n}}{(q)_{2 n+1}}=\prod_{n=1 ; n \neq \pm 3, \pm 4, \pm 7,10(\bmod 20)}^{\infty}\left(1-q^{n}\right)^{-1} .
\end{gathered}
$$

Connor's partition identity related to (7.1) is the following:

THEOREM 7.2. Let $A(n)$ denote the number of partitions of $n$ into parts $\not \equiv 0, \pm 1, \pm 8, \pm 9,10(\bmod 20)$. Let $C(n)$ denote the number of partitions of $n$ into parts where even parts may not be repeated and where odd parts occur only if an even part one unit away occurs. Let $E(n)$ denote the number of partitions of $n$ of the form $b_{1}+b_{2}+\cdots+b_{s}$, where $s$ is even and $b_{1} \geqq b_{2}>$ $b_{3} \geqq b_{4}>b_{5} \geqq \cdots$. Then $A(n)=C(n)=E(n)$. 
We note that the identity

$$
\sum_{n \geqq 0} E(n) q^{n}=\sum_{n \geqq 0} A(n) q^{n}
$$

is rather directly deduced from (7.1); however, the appearance of $C(n)$ in Theorem 7.2 is a complete surprise. While $A(n)$ is related to a partition ideal, neither $C(n)$ nor $E(n)$ is. Identity (7.2) produces a perfect companion for Theorem 7.2. B. Gordon [24] has also proved several interesting theorems of this type.

It might be supposed that we are concluding on a disappointing note. Theorem 4.1 suggests that we may see the beginnings of order amidst chaos, and so the last few paragraphs tend to suggest otherwise. However perhaps a subject seems most vital when (1) general methods are available to begin the attack on numerous problems while (2) other problems and results indicate that many quite unexplored areas remain.

\section{REFERENCES}

(See [8] for an extensive bibliography of this subject.)

1. C. R. Adams, Linear q-difference equations, Bull. Amer. Math. Soc. 37 (1931), 361-400.

2. H. L. Alder, Generalizations of the Rogers-Ramanujan identities, Pacific J. Math. 4 (1954), 161-168. MR 15, 856.

3. G. E. Andrews, On q-difference equations for certain well-poised basic hypergeometric series, Quart. J. Math. Oxford Ser. (2) 19 (1968), 433-447. MR 38 \#6112.

4. - - A new generalization of Schur's second partition theorem, Acta Arith. 14 (1967/68), 429-434. MR 37 \#4042.

5. _- A general theorem on partitions with difference conditions, Amer. J. Math. 91 (1969), 18-24. MR 39 \#4109.

6. - A generalization of the classical partition theorems, Trans. Amer. Math. Soc. 145 (1969), 205-221. MR 40 \#4226.

7. —_, Number theory, Saunders, Philadelphia, Pa., 1971. MR 46 \#8943.

8. - Partition identities, Advances in Math. 9 (1972), 10-51. MR 46 \#5232.

9. - A general theory of identities of the Rogers-Ramanujan type, Amer. Math. Soc. Audio Recording No. 75 and Supplementary Manual, Providence, R.I., 1972.

10. - Summations and transformations of basic Appell series, J. London Math. Soc. (2) 4 (1972), 618-622. MR 46 \#5682.

11. - Sieves for theorems of Euler, Rogers, and Ramanujan, Proc. Western Michigan Conference on Arithmetic Functions, Lecture Notes in Math., no. 251, Springer-Verlag, New York and Berlin, 1972.

12. - Sieves in the theory of partitions, Amer. J. Math. 94 (1972), 1214-1230.

13. - On the Alder polynomials and a new generalization of the Rogers-Ramanujan identities, Trans. Amer. Math. Soc. (to appear).

14. - On the general Rogers-Ramanujan theorem (to appear).

15. - On Rogers-Ramanujan type identities related to the modulus 11, Proc. London Math. Soc. (to appear). 
16. W. N. Bailey, Generalized hypergeometric series, Cambridge Math. Tract, no. 32, Cambridge Univ. Press, Cambridge, 1935.

17. - Some identities in combinatory analysis, Proc. London Math. Soc. (2) 49 (1947), 421-435. MR 9, 263.

18. - Identities of the Rogers-Ramanujan type, Proc. London Math. Soc. (2) 50 (1948), 1-10. MR 9, 585.

19. G. Birkhoff, Lattice theory, Amer. Math. Soc. Colloq. Publ. voi. 25, Amer. Math. Soc., Providence, R.I., 1948, revised 1967.

20. G. D. Birkhoff, The generalized Riemann problem for linear differential equations and the allied problems for linear difference and q-difference equations, Proc. Amer. Acad. Arts and Sci. 49 (1913), 521-568.

21. R. D. Carmichael, The general theory of linear q-difference equations, Amer. J. Math. 34 (1912), 147-168.

22. W. G. Connor, Partition theorems related to some identities of Rogers and Watson, Trans. Amer. Math. Soc. (to appear).

23. G. Frobenius, Über die Charaktere der symmetrischen Gruppe, Sitzber. Preuss. Akad. Berlin 1900, 516-534.

24. B. Gordon, Some continued fractions of the Rogers-Ramanujan type, Duke Math. J. 32 (1965), 741-748. MR 32 \#1477.

25. W. Hahn, Beiträge zur Theorie der Heineschen Reihen. Die 24 Integrale der hypergeometrischen q-Differenzengleichung. Das q-Analogen der Laplace-Transformation, Math. Nachr. 2 (1949), 340-379. MR 11, 720.

26. — Über die höheren Heineschen Reihen und eine einheitliche Theorie der sogenannten speziellen Funktionen, Math. Nachr. 3 (1950), 257-294. MR 12, 711.

27. G. H. Hardy and E. M. Wright, An introduction to the theory of numbers, 4 th ed., Oxford Univ. Press, Oxford, 1960.

28. F. H. Jackson, On basic double hypergeometric functions, Quart. J. Math. Oxford Ser. 13 (1942), 69-82. MR 4, 141.

29. D. E. Knuth, The art of computer programming. Vol. 3. Sorting and searching, Addison-Wesley, Reading, Mass., 1973.

30. F. J. Murray and K. S. Mller, Existence theorems for ordinary differential equations, New York Univ. Press, New York, 1954. MR 16, 358.

31. S. Ramanujan and L. J. Rogers, Proof of certain identities in combinatory analysis, Proc. Cambridge Philos. Soc. 19 (1919), 211-216.

32. L. J. Rogers, On two theorems of combinatory analysis and some allied identities, Proc. London Math. Soc. (2) 16 (1917), 315-336.

33. F. Ryde, A contribution to the theory of linear homogeneous geometric difference equations (q-difference equations), Ph.D. Dissertation, Lund, 1921.

34. I. J. Schur, Zur additiven Zahlentheorie, Sitzler. Akad. Wiss. Berlin Phys.-Math. K1. 1926, 488-495.

35. L. J. Slater, A new proof of Roger's transformations of infinite series, Proc. London Math. Soc. (2) 53 (1951), 460-475. MR 13, 227.

36. —_ Further identities of the Rogers-Ramanujan type, Proc. London Math. Soc. (2) 54 (1952), 147-167. MR 14, 138.

37. —, Generalized hypergeometric functions, Cambridge Univ. Press, Cambridge, 1966. MR 34 \#1570.

Department of Mathematics, Pennsylvania State University, University Park, Pennsylvania 16802 